\title{
Oxygen 15-labeled Oxygen
}

National Cancer Institute

\section{Source}

National Cancer Institute. Oxygen 15-labeled Oxygen. NCI Thesaurus. Code C119710.

A radiopharmaceutical comprised of oxygen-15 labeled oxygen (150-O2) used as a tracer molecule during positron emission tomography (PET). Upon inhalation, 150-O2 binds to hemoglobin in the blood and the labeled red blood cells distribute throughout the vasculature in the body. Upon PET imaging, 150-O2 can be used to determine both the oxygen extraction fraction (OEF) and the metabolic rate of oxygen. 